\title{
Mechanical and Perceptual Analyses of Human Foot Movements in Pedal Operation
}

\author{
Yoshiyuki Tanaka*, Hidekazu Kaneyuki*, Toshio Tsuji*, \\ Tooru Miyazaki ${ }^{\dagger}$, Kazuo Nishikawa ${ }^{\dagger}$ and Takahide Nouzawa ${ }^{\dagger}$ \\ ${ }^{*}$ Graduate School of Engineering, Hiroshima University, Higashi-hiroshima, Japan \\ ${ }^{\dagger}$ Mazda Motor Corporation, Hiroshima, Japan \\ Email: ytanaka@bsys.hiroshima-u.ac.jp
}

\begin{abstract}
A human can maneuver mechanical systems by adjusting his/her own body naturally and effectively according to a target task by utilizing the kinematical and dynamic characteristics of operating systems acquired through sensory organs. If such human sensory and motor characteristics changing in the task can be quantitatively described, it would be useful to design and develop a novel human-machine system so that humans can manipulate a machine more instinctively and comfortably. This paper investigates the interaction between human sensory and motor properties at the foot during the operation of an automobile pedal, as an example of human-machine systems, and demonstrates the close relationship between the perceptual properties of force resistance at the foot and the loads for foot joints much depending on the pedal layout. Finally, based on biomechanical and perceptual analyses, a human-inspired design method of pedal dynamic properties is discussed.

Index Terms-Human-inspired design, human-machine interaction, human force sensation, pedaling motion
\end{abstract}

\section{INTRODUCTION}

A human can maneuver mechanical systems as a part of his/her body by adjusting his/her posture and force according to task conditions. For example, when a driver tries to slow down the speed of an automobile without giving unpleasant inertial force for a fellow passenger, he would control the brake with regulating his foot force while perceiving force-resistance from the pedal by his somatesthesia. Like in such a task with the physical interaction between a human operator and a machine, a human actively regulates his/her dynamic properties of the limbs and senses dynamic properties of the operating interface as well as the external environment. Accordingly, if such human sensory and motor characteristics changing in the operation can be quantitatively described, it would be useful to design and develop a novel human-machine interface so that an operator can manipulate a machine more instinctively and comfortably.

Focusing on an automobile as an example of humanmachine systems, there have been some research studies on the design method of driving interfaces with considering human motor or sensory properties [1]-[7]. For example, Tanaka and Tsuji et al. [1], [2] developed human force manipulability by combining robotic manipulability with human joint-torque characteristics. Their method can evaluate and visualize the spatial characteristics of human force capability at an end-point for the specified posture of the limb. They have applied to the layout design of driving interfaces, such as a pedal, a shifting device and so on. Park and Sheridan [3] discussed an anti-lock brake system by using stiffness of the leg measured in which a subject lay on the horizontal plane to eliminate the effect of gravity. Tanaka et al. [9] proposed a bio-mimetic impedance control method for a pedal-interface system using the database of mechanical impedance properties at the foot measured with respects to leg posture and foot force. However, these researches focused on human motor properties in designing the driving-interface. On the other hand, a few studies reported human perceptual properties at the foot in pedaling. Abbink et al. [5], [6] examined the desirable vibration pattern by the pedal for an alert signal according to the material of shoes from the measured differential threshold of force perception as the foot. Wang et al. [8] evaluated operational feeling of a clutch pedal according to the layout of the pedal and the seat from the biomechanical indices, such as joint-angle, joint-torque and so on. However, they did not discuss the design of pedal layout and resistance with human motor and perceptual properties. Thus, these previous works on the design of driving interfaces did emphasis on human motor or sensory properties although the close relationship between them exists.

The present paper investigates human sensory-motor properties at the foot during pedaling operations and discusses a design methodology of pedal resistances based on experimental results by applying the biological and psychological techniques [9]. This paper is organized as follows: Section II simulates the behavior of contact points on a pedal-pad and a footsole in pedaling according the pedal layout. Based on the simulated results, Section III investigates human perceptual properties to pedal resistances at the foot with the developed experimental system using an impedance-controlled robotic device, and designs the force-stroke characteristics of pedals based on the experimental results as a case study.

\section{Computational Analysis of Pedal Operations}

\section{A. A foot-pedal model}

Observing pedaling movements by the bare foot, the shape of foot fingers as well as the contact conditions changes in the operational angle of a pedal as shown in Fig. 1(a). This paper investigates such behavioral phenomena in pedaling using a simple foot-pedal model constructed with rigid links and rotational joints on the 2D plane as shown in Fig. 1(b). The model assumes that the foot contacts with a pedal at one point 


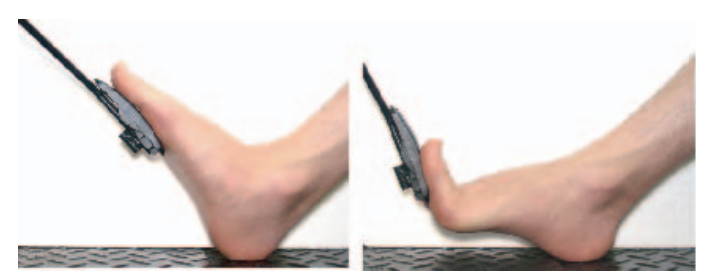

(a)

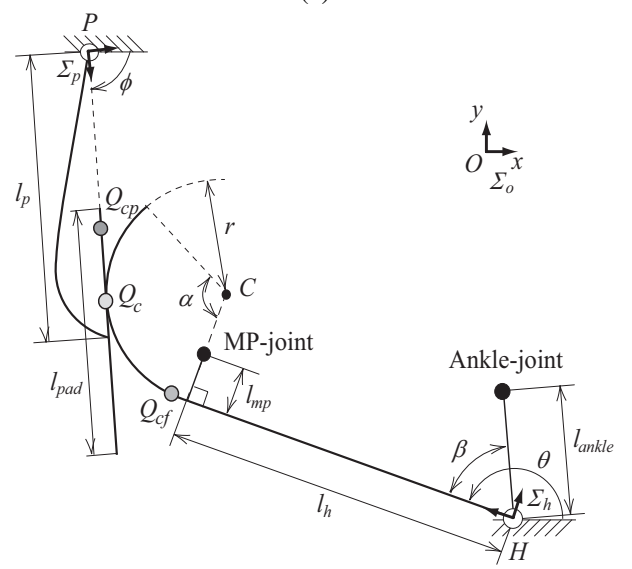

(b)

Fig. 1. A mechanical model of the human foot and the pedal.

TABLE I

PHYSICAL PARAMETERS OF THE BARE-FOOT AND THE MAXIMUM POINT OF THE INDEX $J_{f}$ WITH FOR ALL SUBJECTS.

\begin{tabular}{cccccccc}
\hline Subject & $l_{h}[\mathrm{~mm}]$ & $l_{m p}[\mathrm{~mm}]$ & $l_{\text {ankle }}[\mathrm{mm}]$ & $r[\mathrm{~mm}]$ & $\alpha$ [deg.] & $\beta$ [deg.] & P1 [mm] \\
A & 160 & 20 & 95 & 50 & 110 & 75 & {$[-100,217]$} \\
B & 190 & 25 & 110 & 60 & 110 & 65 & {$[-117,252]$} \\
C & 160 & 20 & 100 & 55 & 110 & 70 & {$[-103,221]$} \\
D & 190 & 25 & 130 & 50 & 110 & 65 & {$[-113,243]$} \\
\hline
\end{tabular}

$Q_{c}$, and expresses the extension posture of foot fingers by the arc part with radius $r$ connected at the metatarsophalangeal (MP) joint. $Q_{c p}$ and $Q_{c f}$ denote the initial contact points on the pedal-pad and the foot-sole at the initial foot posture $\theta_{0}$, respectively. The local coordinate systems, $\Sigma_{p}$ and $\Sigma_{h}$, are set at the root of pedal-arm, $H$, and the tip of heel, $P$, whose positive $x$ directions are direct to $\overrightarrow{P Q_{c p}}$ and $\overrightarrow{H Q_{c f}}$.

The relative distance between the initial contact point $Q_{c}$ and the point $Q_{c p}$ moving on the pedal-pad at the foot-angle $\theta, L_{p}(\theta)$, is given by

$$
\begin{aligned}
L_{p}(\theta) & ={ }^{o} Q_{c}-{ }^{o} Q_{c p} \\
& =\left({ }^{o} H-{ }^{o} P\right)+\left({ }^{o} R_{h}{ }^{h} Q_{h}-{ }^{o} R_{p}{ }^{p} Q_{p}\right)
\end{aligned}
$$

where ${ }^{A} R_{B}(A, B \in\{o, p, h\})$ represents the rotational transformation matrix from the coordinate system $\Sigma_{B}$ to $\Sigma_{A}$. On the other hand, the relative distance for the point $Q_{c f}$ moving

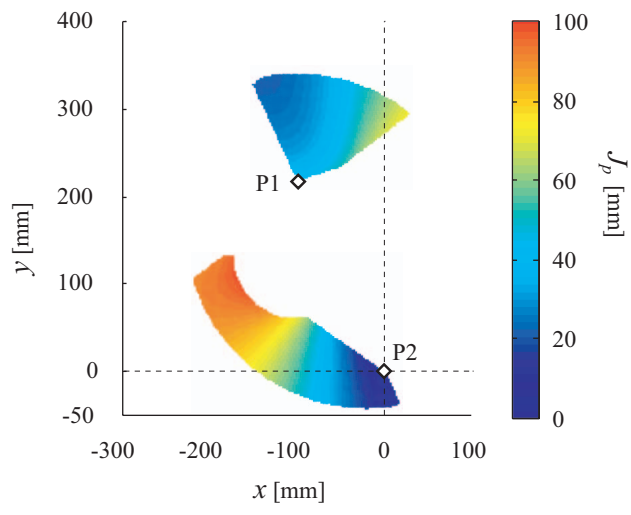

(a) $J_{p}$

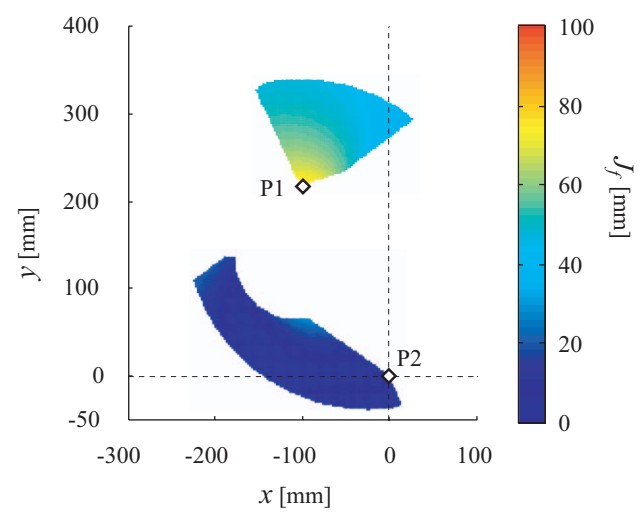

(b) $J_{f}$

Fig. 2. Computed results of the traveled distance of contact points according to the pedal layout.

on the foot-sole at $\theta, L_{f}(\theta)$, can be defined as

$$
L_{f}(\theta)=r \cos ^{-1}\left(\frac{\left({ }^{o} C-{ }^{o} Q_{h}\right) \cdot\left({ }^{o} C-{ }^{o} Q_{f}\right)}{\left\|{ }^{o} C-{ }^{o} Q_{h}\right\|\left\|^{o} C-{ }^{o} Q_{f}\right\|}\right) .
$$

The total traveled distance of the contact point in pedaling, $J_{i}(i \in\{p, f\})$ is then formulated as

$$
J_{i}=\int_{\theta_{0}}^{\theta}\left|\frac{d L_{i}}{d \theta}\right| d \theta .
$$

Note that the difference between $J_{p}(\theta)$ and $J_{f}(\theta)$ suggests the slip on the pedal-pad and the foot-sole would be occurred in pedaling.

\section{B. Influence of the pedal layout}

The influence of the root position of pedal-arm, $P$, on behaviors of the contact points was computationally analyzed using the foot-pedal model by setting the physical properties measured for four healthy subjects (male university students aged 22 - 24), whose data are summarized at Table I.

Fig. 2 shows the simulated results of $J_{p}$ and $J_{f}$ in pedaling from $\theta_{0}=115$ [deg.] to $\theta=145$ [deg.] for Sub. A, where $l_{p}=$ $125[\mathrm{~mm}], l_{\text {pad }}=150[\mathrm{~mm}]$, and the point $H$ is agreed with the origin of $\Sigma_{o}$. As a result of removing the area where pedal 

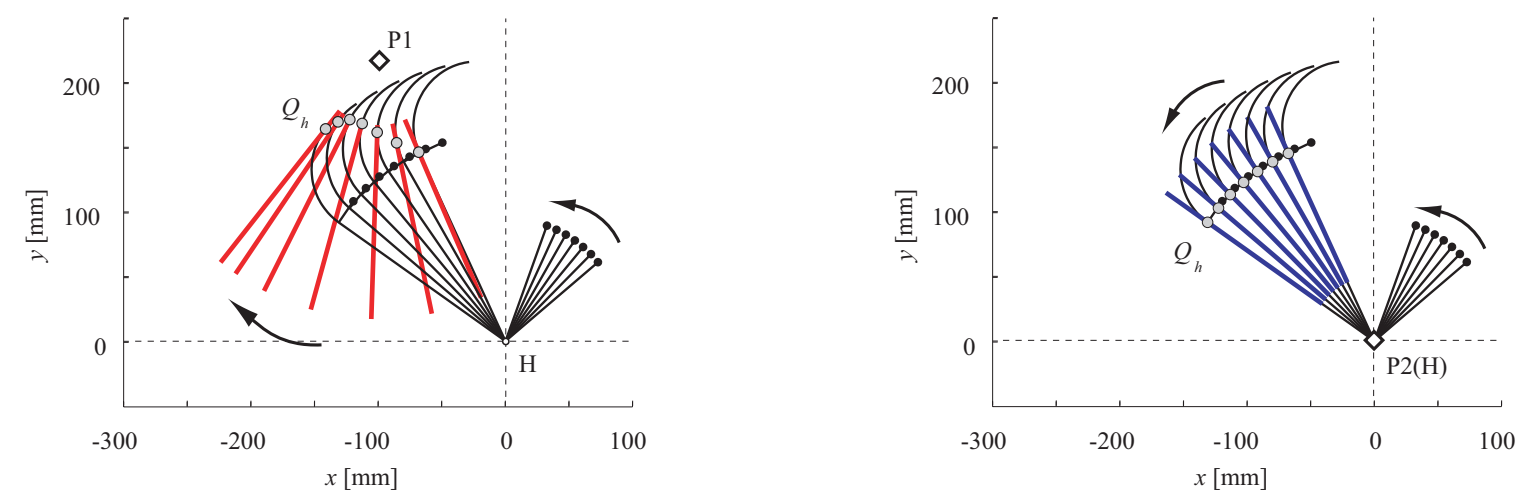

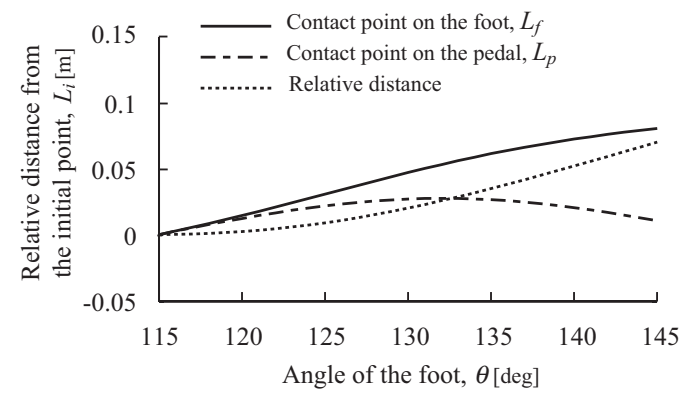

(a)

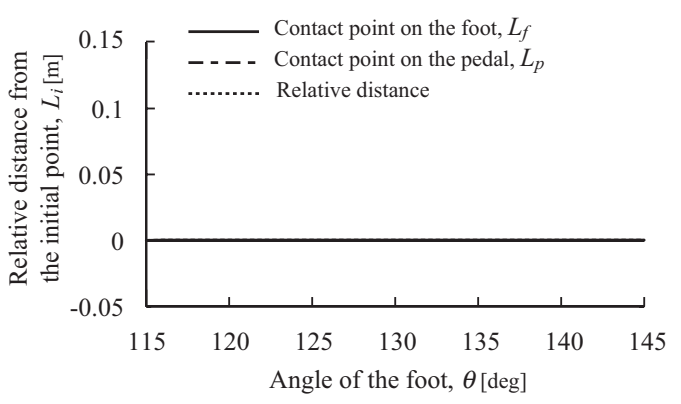

(b)

Fig. 3. Simulated behaviors of foot and pedal according to the roots of pedal-arm (P1 and P2).

operation was impossible, two areas were appeared according to the position of $P$. The index $J_{p}$ increases as the root position $P$ approaching to the heel point $(H)$ in the upper area while decreasing as leaving from $H$ in the lower area. On the other hand, the index $J_{f}$ much changes in $P$ in the upper area while almost stable in the lower area: $J_{f}$ becomes maximum at the point $\mathrm{P} 1$ while minimum at the point $\mathrm{P} 2$ (marked with $\diamond$ in the figure). The calculated positions of P1 and P2 for all subjects are in Table I.

Fig. 3 shows a set of stick pictures of the foot-pedal model and the corresponding behaviors of contact points during pedal operations at $\mathrm{P} 1$ and $\mathrm{P} 2$. The contact point $Q_{c}$ moves to the tip of foot as increasing the foot angle $\theta$ in the case at P1 while keeps to the initial contact point $Q_{c f}$ at P2. These results suggest that operational feeling in pedaling would much depend on the position of $P$ because of contact conditions. The next section investigates how such contact conditions would affect human perception to force resistances at the foot.

\section{INFLUence of PedAl LAyout into ForCe PERCEPTION PROPERTIES}

A set of force perception tests at the foot was carried out by means of the psychological technique based on a magnitude estimate method [10] for the two points of pedal rotational center P1 and P2 $(\mathrm{H})$, i.e., the point where $J_{f}$ was maximum or minimum.

\section{A. Method}

Fig. 4 shows a schematic view of an experimental system developed for a perceptual test by the leg in this paper. The system is composed of a direct-drive rotary motor (NIPPON THOMPSON CO., LTD., RM1A) at which a pedal-arm with flat pad is attached on the rotational center of the motor, a computer for motor control and signal processing, and a biofeedback display to provide a rotation angle of the pedal $\phi$ to a subject in on-line. The operational force $F$ generated by the subject is measured by a six-axis force/torque sensor (BL AUTOTEC LTD., FT 30/300HSS) attached on the pad and $\phi$ is measured by a built-in encoder of the motor.

An impedance control method [11] is applied into the motor control so that various force loads can be provided to the subject's foot by adjusting robot impedance parameters in the following dynamics of the pedal as

$$
\tau(t)=M \ddot{\phi}(t)+B \dot{\phi}(t)+K\left(\phi(t)-\phi_{e}(t)\right),
$$

where $M, B(\phi)$ and $K(\phi)$ are the pedal inertia, viscosity and stiffness, respectively, and the angle $\phi_{e}$ is the equilibrium of $K$. The desired magnitude of force stimulus was produced by regulating the pedal stiffness $K$ under that the inertia was set at $M=0.03\left[\mathrm{kgm}^{2}\right]$ and the viscosity $B$ was automatically adjusted to set the damping coefficient of the pedal system at $\zeta=1.2$ to realize steady pedal operations. Stable control of the experimental system and high-quality data measurement attained in sampling frequency $1[\mathrm{kHz}]$ by using a DSP board (dSpace: DS1104). 


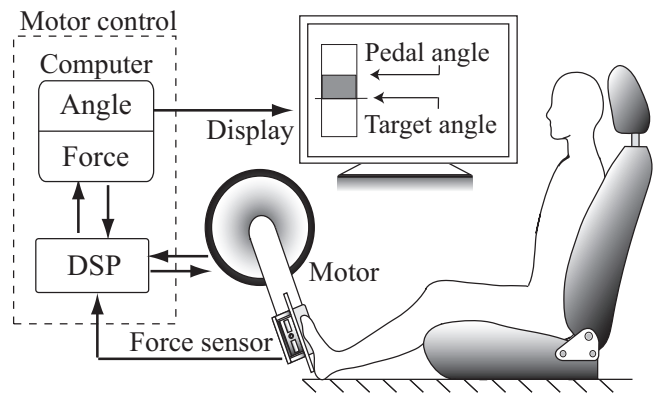

(a)

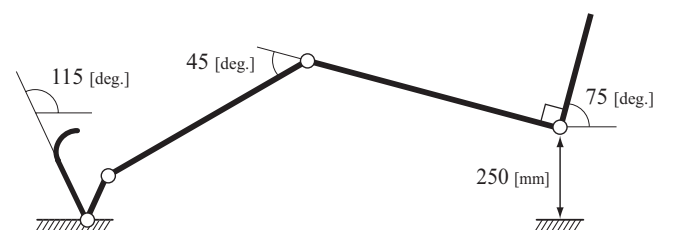

(b)
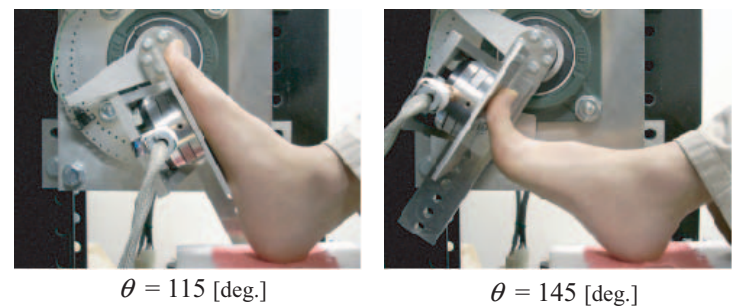

(c)

Fig. 4. Experimental apparatus and conditions designed for a force perception test at the foot.

In the experiment, a subject placed his bare foot on the pedal-pad under the specified posture of his lower extremity as shown in Fig. 4(b) and kept the heel point as much as possible. The subject was then instructed to memorize the standard force stimulus $F_{s}=20[\mathrm{~N}]$ provided from the pedal at the initial foot angle $\theta=115$ [deg.] and perceive a comparison stimuli $F_{r}(=5$, $10,15,20,25,30,35,40[\mathrm{~N}])$ for five seconds at the specified foot angles $\theta=115,145$ [deg.] (See Fig 4(c)). He then orally reported a ratio of the perceived force magnitude $F_{p}$ to the standard one $F_{s}$ by percentage terms. Each of comparison forces was provided at five times after a short preliminary test, where the force magnitude was randomly selected. The target pedal-angle corresponding to the specified foot-angle was confirmed at the preliminary test in each subject.

\section{B. Results}

Fig. 5 shows typical results of the perception tests at the points $\mathrm{P} 1$ and $\mathrm{P} 2$ for Sub. B, where the vertical axis is the magnitude of perceived force and the horizontal axis is that of true force normalized with $F_{s}(=20[\mathrm{~N}])$; red circle and blue rectangle are the data measured under $\theta=115,145$ [deg.], respectively; and the logarithmic regression curve was

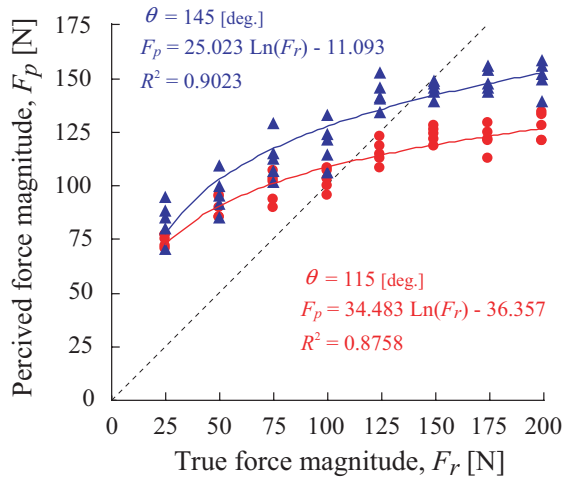

(a)

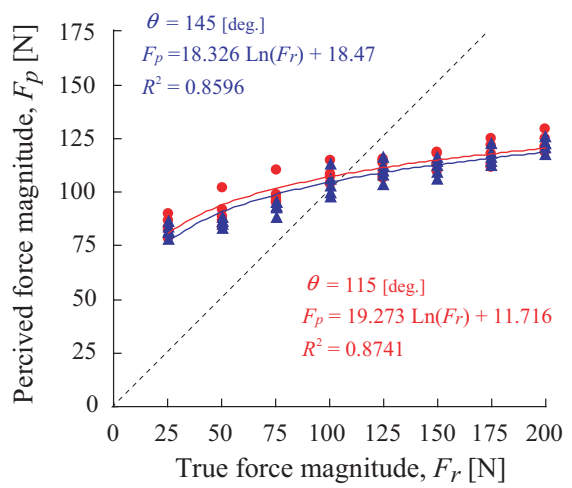

(b)

Fig. 5. An example of the experimental results depending on foot-angle and pedal-layout for Sub. B.

calculated by the least squares method with the data measured at each foot-angle.

With regardless of the point of pedal rotational center, the subject tended to feel the magnitude of a provided force as larger than that of an actual force in which $F_{r}$ was smaller than $F_{s}$, and vise versa. In addition, the coefficient of determination is over 0.84 and the perceived value is almost proportional to the logarithm of the comparison stimuli $F_{r}$. These indicate that the Weber-Fechner law [10] is almost satisfied in force perception at the foot within the designed experimental conditions. The almost same characteristics were observed for all subjects. Table II summarizes the parameters of regression curves and the coefficient of determination for the all subjects. Why did the subjects feel such perceptual differences between the root points to the same set of force magnitudes?

Recalling the computed results in the previous section, the contact point $Q_{c f}$ travels from the initial position to the tip of toe as increasing the foot angle $\theta$ in the case for P1. This positional change of $Q_{c f}$ naturally leads to that a major part (joint) receiving force-resistance changes according to the foot angle. Fig. 6 shows the changes of joint-torque equivalent to the force-resistance $F_{r}=20[\mathrm{~N}]$ calculated using the footpedal model with the physical parameters for Sub. B. The 
TABLE II

ESTIMATED PARAMETERS OF THE LOGARITHMIC REGRESSION CURVE AND THE COEFFICIENT OF DETERMINATION

\begin{tabular}{|c|c|c|c|c|c|c|}
\hline P1 & \multicolumn{3}{|c|}{115 [deg.] } & \multicolumn{3}{|c|}{145 [deg.] } \\
\hline Subject & $a$ & $b$ & $R^{2}$ & $a$ & $b$ & $R^{2}$ \\
\hline A & 44.38 & -107.32 & 0.84 & 49.64 & -115.11 & 0.90 \\
\hline B & 25.04 & -11.53 & 0.91 & 35.19 & -40.12 & 0.87 \\
\hline $\mathrm{C}$ & 58.83 & -163.15 & 0.91 & 70.61 & -205.99 & 0.92 \\
\hline D & 58.14 & -170.38 & 0.91 & 66.55 & -198.47 & 0.94 \\
\hline P2 & \multicolumn{3}{|c|}{115 [deg.] } & \multicolumn{3}{|c|}{145 [deg.] } \\
\hline Subject & $a$ & $b$ & $R^{2}$ & $a$ & $b$ & $R^{2}$ \\
\hline A & 47.94 & -120.82 & 0.92 & 39.98 & -90.32 & 0.93 \\
\hline B & 18.66 & -16.67 & 0.87 & 19.43 & -10.93 & 0.88 \\
\hline $\mathrm{C}$ & 51.17 & -128.76 & 0.96 & 51.54 & -126.30 & 0.90 \\
\hline $\mathrm{D}$ & 46.46 & -120.99 & 0.91 & 52.15 & -136.08 & 0.96 \\
\hline
\end{tabular}

results demonstrate that joint-torque decreases at the ankle in proportional to $\theta$ while increases at the MP where the rotational center is positioned at $\mathrm{P} 1$; i.e., a major joint contributing for the pedal operation changes from the ankle-joint to the MP-joint.

Fig. 7 shows the relationship between the change of contributed joint-torque $J_{\tau}$ calculated using the simple foot-pedal model (Fig. 1(b)) and the perceptual differences between the foot angles $J_{F}$ defined by the following equation as:

$$
J_{F}=\int_{25}^{200}\left\{\left.F_{p}\left(F_{r}\right)\right|_{\theta=145}-\left.F_{p}\left(F_{r}\right)\right|_{\theta=115}\right\} d F_{r},
$$

where $F_{P}\left(F_{r}\right)$ represents the regression curve obtained by the experiments. The significant differences between the root points of pedal-arm was observed at $1 \%$ significant level. It can be seen that perceptual properties change depending on which joint mainly contributed for generating the reaction force at the contact point. It has bee reported that a human feels a force magnitude larger when he perceives by a smaller muscle than a larger muscle [12], [13]. The agonists of the ankle joint (gastrocnemius, soleus) are much larger than those of the MP joint (flexor hallucis longus, flexor hallucis brevis, lumbricales, flexor digitorum longus, flexor digitorum brevis) [14], [15]. Consequently, the subjects felt force stimuli as much larger for $\theta=145$ [deg.] at the point P1 where the torque load to the MP joint is larger than the ankle joint.

\section{Application}

The experimental results demonstrated that human force perception at the foot depends on the pedal layout, i.e., the contact condition between foot and pedal. Paradoxically, even which a comfortable dynamics around the rotational axis of the pedal could be designed for a human driver, it would much change depending on the pedal layout. Based on this point, a human-inspired design approach of pedal dynamics is

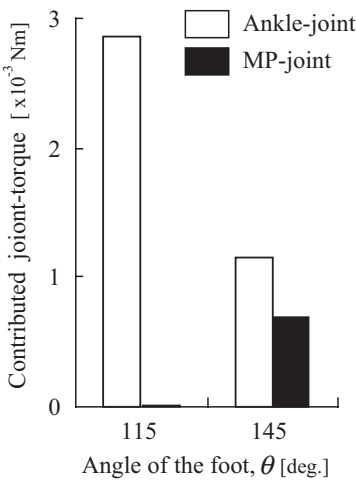

(a)

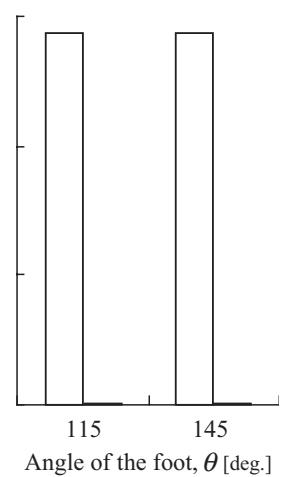

(b)
Fig. 6. Computed joint-torque for the specified two foot-angle at P1 and P2 for Sub. B.

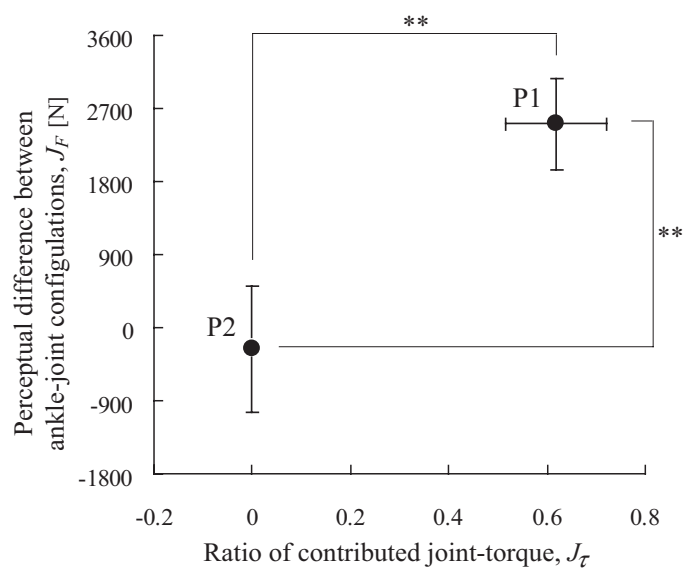

Fig. 7. Relationship between the rate of contributed joint-torque and the perceptual difference.

discussed. The target problem set in this paper is to find the force-stroke properties of a pedal at the contact point $F_{d}(\phi)$ so that an operator would feel the designed resistance properties $F_{p d}(\phi)$ at the foot without respect to the root point $P$.

Fig. 8(a) shows the proposed design procedure of pedal force-resistance considering the observed perception properties. $F_{d}$ is defined with $F_{p d}$ by

$$
F_{d}(\phi)=\exp \left(\frac{F_{p d}(\phi)-b}{a}\right)
$$

where the parameters $a, b$ are the values of a logarithmic curve calculated with measured perceptual data for all foot postures at each layout of the root of pedal-arm under assumption that the perceptual properties were preserved for different ranges of force stimuli. Note that $F_{p d}$ functions as a compensator of the perceptual changes according to the root layout.

Fig. 8(b) shows an example of the designed force-stroke properties $F_{d}(\phi)$ to the desired properties $F_{r}(\phi)=\frac{20}{45}(\phi-$ $65)+10[\mathrm{~N}]$ perceiving at the points $\mathrm{P} 1$ and $\mathrm{P} 2$, where $a$ and $b$ were set at the mean values of the data measured at 
each point for all the subjects presented in Table II. The shade zones represent the individual differences of perceiving forceresistance which was calculated back using the parameters of logarithmic curves in each subject. The designed force $F_{d}$ is modulated from $F_{p d}$ with respects to the pedal-angle $\phi$ and the pedal layout, smaller force for the small angle while larger force for the larger angle, so that the human operator would feel the same magnitude of resistance $F_{d}$ in pedaling even at the different pedal layouts.

The results suggest that a pedal devise can give a human the desired operational feeling even for different pedal layouts and/or mechanisms when dynamic properties of the pedal were designed with well-considering such human force-perceptual properties at the foot changing in the contact condition.

\section{CONCLUSION}

This paper first analyzed the contact conditions between foot-sole and pedal-pad in pedaling by using the simple footpedal model and showed that the contact points on foot-sole and pedal-pad much depend on the root point of pedal-arm. Based on the computed results, force perception tests at the foot were carried out with the four subjects in the different two pedal layouts using the developed experimental system for pedal operations. The perceptual results demonstrated that human force perception characteristics at the foot change in the pedal payout even where the same magnitude of force stimulus were provided. Such an observed phenomenon was then explained from biomechanical and psychological viewpoints. Finally, as one of potential applications of the research results, the mechanical and perceptual design methodology of pedal dynamics was discussed.

Future research will be directed to perform further perceptual tests under other experimental conditions, such as leg configurations and the effects of shoe's material, and to build a more detail foot-pedal model, while applying the proposed human-inspired design methodology to other human-machine systems.

\section{ACKNOWLEDGMENT}

This research work was supported in part by a Grant-inAid for Scientific Research from the Japanese Ministry of Education, Science and Culture (18760193).

\section{REFERENCES}

[1] Y. Tanaka, N. Yamada, I. Masamori, and T. Tsuji: "Manipulability analysis of lower extremities based on human joint-torque characteristics," Proceedings of the 2nd International Symposium on Measurement, Analysis and Modeling of Human Functions, pp. 261-266, 2004.

[2] Y. Tanaka, N. Yamada, K. Nishikawa, I. Masamori, and T. Tsuji: "Manipulability analysis of human arm movements during the operation of a variable-impedance controlled robot," Proceedings of the 2005 IEEE/RSJ International Conference on Intelligent Robotics and Systems, pp. 3543-3548, 2005.

[3] S. Park and T. B. Sheridan: "Enhanced human-machine interface in braking," IEEE Transactions on systems, man, and cybernetics-Part A, Vol. 34, pp. 615-629,2004.

[4] Y. Tanaka, T. Onishi, T. Tsuji, N. Yamada, Y. Takeda, and I. Masamori: "Analysis and modeling of human impedance properties for designing a human-machine control system," Proceedings of the 2007 IEEE International Conference on Robotics and Automation, pp. 3627-3632, 2007.

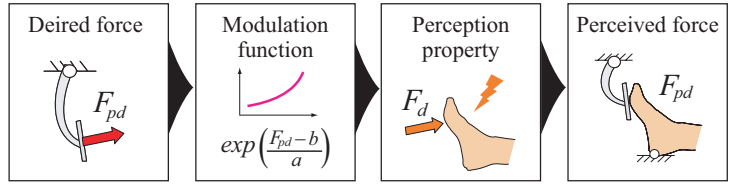

(a)

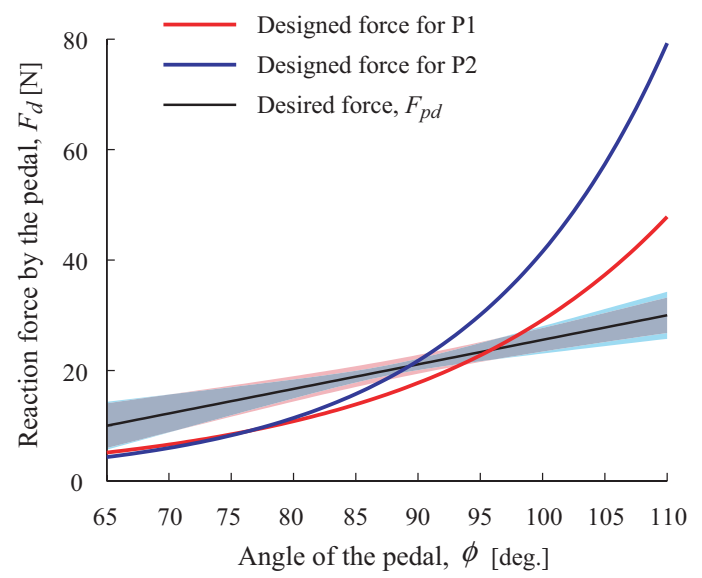

(b)

Fig. 8. Human-inspired design of the force resistance at the foot for a pedaling device.

[5] D. A. Abbink and V. D. Helm: "Force perception measurements at the foot," Proceedings of the 2004 IEEE International Conference on Systems, Man and Cybernetics, Vol.3, pp. 2525-2529, 2004.

[6] D. A. Abbink, V. D. Helm, and E. R. Boer: "Admittance measurements of the foot during 'maintain position' and 'relax' tasks on a gas pedal,'Proceedings of the 2004 IEEE International Conference on Systems, Man and Cybernetics, Vol.3, pp. 2519-2524, 2004.

[7] M. Mulder, M. M. V. Paassen, S. Kitazaki, S. Hijikata, and E. R. Boer: "Car-following support with haptic gas pedal feedback", Proceedings of IFAC Symposium on Analysis, Design, and Evaluation of HumanMachine Systems, 2004.

[8] X. Wang, B. L. Breton-Gadegbeku, and L. Bouzon: "Biomechanical evaluation of the comfort of automobile clutch pedal operation," International Journal of Industrial Ergonomics, Vol. 34, Issue 3, pp. 209-221, 2004.

[9] Y. Tanaka and T. Tsuji: "Directional properties of human hand force perception in the maintenace of arm posture," Neural Information Processing (14th International Conference, ICONIP 2007), pp. 933-942, Springer Berlin/Heidelberg, 2008.

[10] S. S. Stevens: "Psychophysics: Introduction to its perceptual, neural, and social prospects," Ed. G. Stenens, New York: John Wiley, 1975.

[11] N. Hogan: "Impedance control: an approach to manipulation: Parts I, II, III,” ASME Journal of Dynamic Systems, Measurement, and Control, Vol. 107, no. 1, pp. 1-24, 1985.

[12] L. A. Jones: "Role of central and peripheral signals in force sensation during fatigue," Experimental Neurology, Vol. 81, pp. 497-503, 1983.

[13] L. A. Jones: "Perceptual constancy and the perceived magnitude of muscle forces," Experimental Brain Research, Vol. 151, pp. 197-203, 2003.

[14] H. Kura, Z. P. Luo, H. B. Kitaoka, and K. N. An: "Quantitative analysis of the intrinsic muscles of the foot," The Anatomical Record, Vol. 249, Issue 1, pp. 143-151, 1998.

[15] M. D. Klein Horsman, H. F. Koopman, F. C. van der Helm, L. P. Prosé, and H. E. Veeger: "Morphological muscle and joint parameters for musculoskeletal modelling of the lower extremity," Clinical Biomechanics, Vol. 22, Issue 2, pp. 239-247, 2007. 